\title{
NOUVELLE
}

\section{Voie de signalisation Notch et développement précoce des mammifères}

Sarah Cormier, Céline Souilhol, Charles Babinet, Michel Cohen-Tannoudji

Unité de Génétique fonctionnelle de la souris, CNRS URA 2578, Institut Pasteur, 25, rue du Docteur Roux, 75724 Paris Cedex 15, France.

m-cohen@pasteur.fr
> Pendant la première partie du développement, l'embryon de mammifère est le siège d'un grand nombre d'événements génétiques, épigénétiques et morphogénétiques qui le préparent d'une part à son implantation dans l'utérus, dont dépendra son approvisionnement nutritif et énergétique ultérieur, et d'autre part à la période de prolifération et de migration cellulaires intenses qui accompagne la mise en place des feuillets embryonnaires et des axes définitifs de l'embryon pendant la gastrulation. Avant l'implantation, l'embryon, appelé blastocyste, est composé de trois types cellulaires:

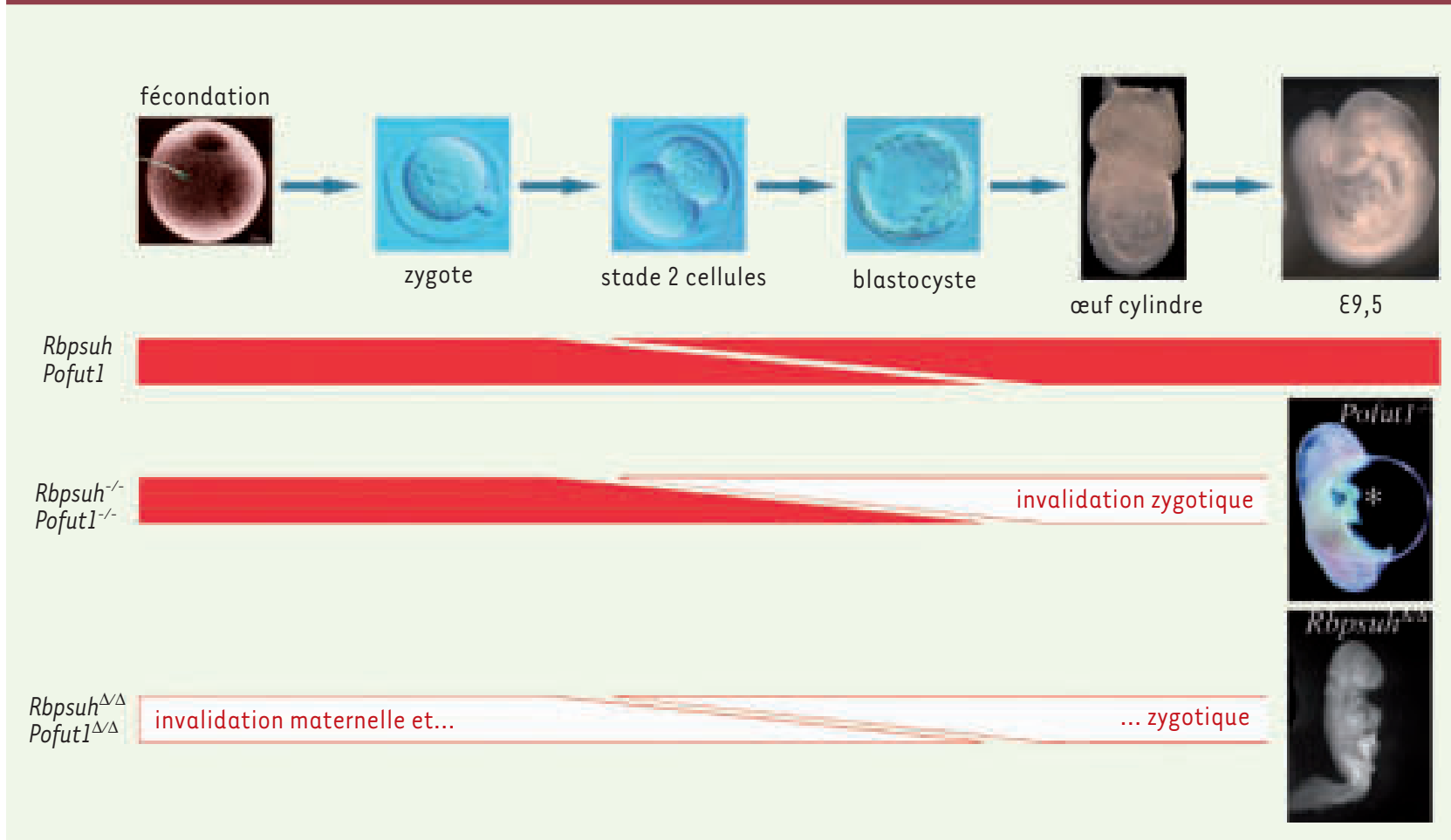

Figure 1. La voie de signalisation Notch n'est pas indispensable pour le développement pré-implantatoire de l'embryon. Les gènes Rbpsuh et Pofutl, codant tous les deux pour des acteurs centraux de la voie de signalisation Notch, sont exprimés dans l'ovocyte et tout au long du développement embryonnaire (schématisé par les flèches rouges). L'invalidation «classique » de l'un ou l'autre de ces deux gènes entraîne l'élimination des transcrits zygotiques (schématisée par les flèches blanches) ; les embryons «knock-out » correspondants, notés Rbpsuh ${ }^{-/-}$et Pofut ${ }^{{ }^{-/-}}$, se développent normalement jusqu'à la fin de la gastrulation puis présentent de nombreux défauts avant de mourir vers le dixième jour de développement. Ces observations indiquent que l'activité zygotique de la voie Notch n'est pas essentielle pour le développement pré-implantatoire de l'embryon. Cependant, elle laisse entière la possibilité d'une fonctionnalité plus précoce de cette voie via l'expression maternelle des gènes impliqués dans celle-ci. L'élimination simultanée des transcrits maternels et zygotiques des gènes Rbpsuh et Pofutl a pu être obtenue grâce à une approche de mutagenèse conditionnelle. Le phénotype de létalité embryonnaire des embryons correspondants, notés $R b p s u h^{\Delta / \Delta}$ et Pofutl ${ }^{\Delta / \Delta}$, est en tout point semblable à celui des embryons $R_{b p s u h^{-/-}}$et Pofut $\mathrm{I}^{-/-}$. Ces observations démontrent clairement que la voie de signalisation Notch n'est pas indispensable pour le développement pré-implantatoire chez la souris. 
la masse cellulaire interne $(\mathrm{MCl})$ qui sera à l'origine du futur embryon, le trophectoderme, futur placenta, et l'endoderme primitif qui constituera avec le mésoderme extraembryonnaire le futur sac vitellin. L'établissement de ces lignages est finement régulé et plusieurs mécanismes expliquant l'apparition et le maintien des premières différenciations cellulaires ont été proposés [1].

Chez les métazoaires, la voie de signalisation Notch est un mode fondamental de communication entre cellules voisines [2, 3]. Elle est fréquemment impliquée dans des choix de différenciations cellulaires via le processus d'inhibition latérale qui limite la propagation d'un type de différenciation ou via l'induction latérale qui homogénéise les choix de destinées au sein d'une population. II n'est alors pas étonnant de constater que la voie Notch

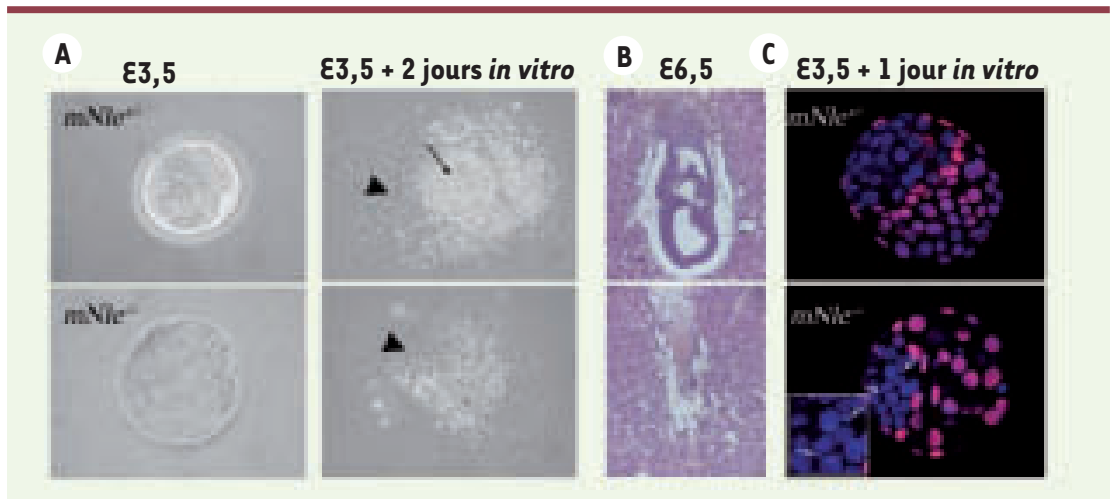

Figure 2. Le gène mouse Notchless (mNle) est nécessaire pour la survie des cellules de la masse interne du blastocyste. A. Culture d'embryons provenant de croisements entre souris $\mathrm{mNle} \mathrm{e}^{+/-}$et prélevés au stade blastocyste (3,5 jours de développement ou $\varepsilon 3,5)$. Après deux jours de culture, les cellules de la masse interne des blastocystes $\mathrm{mNle} e^{+/+}$et $\mathrm{mNl} e^{+/-}$prolifèrent (flèche) audessus des cellules du trophectoderme qui se différencient et s'attachent au fond de la boîte de culture (tête de flèche). À 3,5 jours, les blastocystes $m N^{-/ /}$ne sont pas différents des blastocystes témoins. Après deux jours de culture, les cellules du trophectoderme se différencient et $s^{\prime}$ attachent au fond de la boîte alors que les cellules de la masse interne dégénèrent rapidement. B. Coupes histologiques d'une corne utérine provenant d'une femelle $m \mathrm{Nle}^{+/-} 6,5$ jours après avoir été accouplée avec un mâle $m N / e^{+/-}$. En haut : site d'implantation normal dans lequel on distingue clairement les différentes structures embryonnaires et extra-embryonnaires. En bas: site d'implantation anormal dans lequel on ne distingue plus de structures embryonnaires. Ce type de site d'implantation résulte très probablement de la nidation d'un embryon $\mathrm{mNle} e^{-/-}$suivi de sa rapide dégénérescence. $C$. Les cellules de la masse interne des embryons déficients pour mNle meurent par apoptose. Vingt-quatre heures avant l'apparition du phénotype en culture et grâce à une coloration de I'ADN au DAPI (coloration bleue), on observe chez les embryons $\mathrm{mNle} \mathrm{e}^{-/-}$une nette augmentation de cellules apoptotiques (noyaux fragmentés signalés par les flèches) dans la masse cellulaire interne. À ce stade, la protéine nucléaire CDX2 est exprimée spécifiquement dans le trophectoderme (coloration rouge). différenciation et le développement de nombreux tissus comme les somites, les vaisseaux, le système nerveux central et bien d'autres encore [5]. Les défauts les plus précoces observés chez ces embryons mutants concernent la mise en place de l'asymétrie droite-gauche suite à des anomalies d'expression du gène Nodal - une des cibles directes de la voie Notch - dans le nœud vers le $8^{e}$ jour de développement [6]. L'absence de défauts avant et/ou pendant la gastrulation chez ces mutants contraste avec des données récentes qui montrent que, dans différents modèles de différenciation in vitro de cellules souches embryonnaires murines et humaines (cellules ES qui dérivent de la $\mathrm{MCl}$ du blastocyste), la voie Notch joue un rôle essentiel dans le choix entre différenciation neuronale et différenciation mésodermique $[7,8]$. Ainsi, l'absence de défauts importants de spécification des feuillets embryonnaires chez les embryons mutants montre que le rôle de la voie Notch dans ce processus est plus critique ex vivo qu'in vivo. L'ensemble de ces observations suggèrent fortement que l'embryon de souris est capable, en partie du moins, de compenser in vivo les défauts engendrés par le dérèglement de la voie de signalisation Notch.

À première vue, la voie Notch ne semble pas jouer un rôle critique avant la gastrulation et au cours du développement préimplantatoire. En effet, non seulement des blastocystes normaux sont formés en l'absence de tel ou tel gène de la voie Notch, mais des mutations perte et gain de fonction de la voie Notch dans les cellules ES sont sans conséquence sur leur maintien, leur auto-renouvellement ou leur survie $[7,9]$. Comment expliquer alors que les gènes codant pour les principaux acteurs de cette voie sont exprimés (certains de façon dynamique) dans l'ovocyte et/ou l'embryon pré-implantatoire $[10,11]$ ? Une première possibilité résulte de la présence de transcrits ovocytaires qui masqueraient, chez des embryons mutants pour des gènes de la voie Notch, un rôle éventuel de cette 
voie dans l'embryon précoce. En effet, chez les embryons knock-out, l'apport de stocks maternels peut suppléer l'absence d'expression zygotique et contribuer ainsi au bon déroulement du développement précoce. Afin d'examiner cette éventualité, des embryons totalement déficients pour la voie Notch ont été produits par deux équipes indépendantes (dont la nôtre) en inactivant à la fois dans l'ovocyte et dans le zygote deux gènes essentiels au fonctionnement normal de celle-ci: Rbpsuh codant pour l'unique facteur de transcription de la voie, RBP-JK - qui s'associe aux domaines intracytoplasmiques activés des quatre récepteurs Notch, et représente donc un élément clé du contrôle de la transcription des gènes cibles de Notch [12] - et Pofut1 (protein 0-fucosyltransferase 1) codant pour une enzyme qui catalyse une modification post-traductionnelle du récepteur Notch indispensable pour son activation par un ligand. La létalité de ces embryons ne diffère en rien de celle des embryons knock-out classiques correspondants $[13,14]$, renforçant ainsi la notion que la voie Notch n'est pas indispensable pour le développement préimplantatoire chez la souris (Figure 1).

Dans ce contexte, le phénotype de létalité péri-implantatoire des souris mutantes pour Brainiac et mNotchless est tout à fait intrigant $[15,16]$. En effet, Brainiac, qui code pour une glycosyltranférase, interagit génétiquement avec la voie Notch chez le nématode et la mouche $[17,18]$. Le gène Notchless quant à lui a été initialement identifié chez la drosophile lors d'un crible suppresseur du phénotype d'aile crénelée, trait caractéristique d'une perte d'activité de la voie Notch chez cette espèce $(\rightarrow)$. La protéine Notchless lie la partie intracellulaire du

récepteur Notch $(\rightarrow)$ Voir Figure 1 page 21 et, par un méca- de ce numéro nisme qui reste à élucider, module positivement ou négativement la signalisation Notch en fonction du contexte cellulaire [19]. II est donc probable que l'apoptose sélective des cellules de la $\mathrm{MCl}$ et la mort des embryons de souris déficients pour motchless juste après l'implantation (Figure 2) [15] résultent d'un dérèglement de l'activité de la voie Notch très précocement. Sur la base des expériences décrites dans le paragraphe précédent, il semble exclu que cette létalité soit consécutive à une perte d'activité. En revanche, nos observations préliminaires indiquent que la suractivation de la voie Notch pendant le développement pré-implantatoire provoque un phénotype similaire à celui des embryons déficients pour mNotchless. Si ces résultats se confirmaient, il serait tentant de réconcilier l'ensemble de ces observations à l'aide du modèle suivant. Avant l'implantation, les différents composants sont en place et la voie Notch est active (ou activable). Le rôle joué par la voie Notch pendant cette période, s'il existe, peut être pris en charge par un mécanisme compensatoire en cas de défaillance. Au moment de l'implantation, les produits de certains gènes comme mNotchless agiraient pour maintenir l'activité de la voie Notch en deçà d'un niveau délétère pour la survie de l'embryon. Plus tard, la levée de cette inhibition permettrait la mobilisation rapide de la voie de signalisation pendant la gastrulation. Des travaux complémentaires, notamment sur le mécanisme d'action de mNotchless, permettront d'éprouver la véracité de ce modèle. $\diamond$

Notch signalling pathway and early mammalian embryogenesis

\section{RÉFÉRENCES}

1. Yamanaka Y, Ralston A, Stephenson RO, Rossant J. Cell and molecular regulation of the mouse blastocyst. Dev Dyn 2006 ; 235 : 2301-14.
2. Artavanis-Tsakonas $S$, Matsuno K, Fortini ME. Notch signaling. Science $1995 ; 268: 225-32$.

3. Schweisguth F. Notch signaling activity. Curr Biol 2004 ; $14:$ R129-38.

4. Shi S, Stanley P. Evolutionary origins of Notch signaling in early development. Cell Cycle $2006 ; 5$ : 274-8.

5. Yoon K, Gaiano N. Notch signaling in the mammalian central nervous system: insights from mouse mutants. Nat Neurosci $2005 ; 8: 709-15$.

6. Vincent $S$. Left-right asymmetry: Notch acts upstream of Nodal. Med Sci (Paris) 2003; 19: 1188-90.

7. Lowell S, Benchoua A, Heavey B, Smith AG. Notch promotes neural lineage entry by pluripotent embryonic stem cells. PLoS Biol 2006; 4 : el21.

8. Nemir M, Croquelois A, Pedrazzini T, Radtke F. Induction of cardiogenesis in embryonic stem cells via downregulation of Notchl signaling. Circ Res 2006; 98: 1471-8.

9. Noggle SA, Weiler D, Condie BG. Notch signaling is inactive but inducible in human embryonic stem cells. Stem Cells 2006 ; 24 : 1646-53.

10. Cormier S, Vandormael-Pournin S, Babinet C, Cohen-Tannoudji M. Developmental expression of the Notch signaling pathway genes during mouse preimplantation development. Gene Expr Patterns $2004 ; 4: 713-7$.

11. Wang QT, Piotrowska K, Ciemerych MA, et al. A genomewide study of gene activity reveals developmental signaling pathways in the preimplantation mouse embryo. Dev Cell 2004 ; 6 : 133-44.

12. Brou $C$, Logeat F. Endocytose et voie de signalisation Notch. Med Sci (Paris) 2006 ; 22 : 685-8.

13. Shi S, Stahl M, Lu L, Stanley P. Canonical Notch signaling is dispensable for early cell fate specifications in mammals. Mol Cell Biol 2005; 25: 9503-8.

14. Souilhol C, Cormier S, Tanigaki K, Babinet C, CohenTannoudji, M. RBP-J(kappa)-dependent Notch signaling is dispensable for mouse early embryonic development. Mol Cell Biol 2006 ; 26 : 4769-74.

15. Comier S, Lebras S, Vandormael-Pournin S, et al. The murine Notchless protein is essential for survival of embryos and maintenance of inner cell mass. Mol Cell Biol 2006 ; 26 : 3541-9.

16. Vollrath B, Fitzgerald KJ, Leder P. A murine homologue of the Drosophila brainiac gene shows homology to glycosyltransferases and is required for preimplantation development of the mouse. Mol Cell Biol $2001 ; 21$ : 5688-97.

17. Goode S, Melnick M, Chou TB, Perrimon N. The neurogenic genes egghead and brainiac define a novel signaling pathway essential for epithelial morphogenesis during Drosophila oogenesis. Development 1996 ; 122 : 3863-79.

18. Katic I, Vallier LG, Greenwald I. New positive regulators of lin-12 activity in Caenorhabditis elegans include the BRE-5/Brainiac glycosphingolipid biosynthesis enzyme. Genetics $2005 ; 171: 1605-15$.

19. Royet J, Bouwmeester T, Cohen SM. Notchless encodes a novel WD40-repeat-containing protein that modulates Notch signaling activity. EMBO 1998 ; $17: 7351-60$.

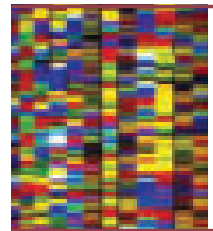

Retrouvez chaque mois médecine/sciences sur France-Info dans la chronique «Info-Sciences 》 de Marie-Odile Monchicourt, du lundi au mercredi. france-info.com 